\title{
Quality and Safety Traceability System of Aquatic Products Based on Internet of Things
}

\author{
https://doi.org/10.3991/ijoe.v13i09.7590 \\ Xiao-hua $\mathrm{Xu}$ \\ Tianjin Agricultural University, Tianjin, China \\ xuxiaohua@tjau.edu.cn
}

\begin{abstract}
In order to better detect the quality of aquatic products, a water quality and safety traceability system based on Internet of things has been designed. By analyzing the marine product quality and safety control, a standardized, intelligent and universal marine product quality safety traceability system is proposed based on the Internet of things technology and the characteristics of the seafood industry chain are analyzed. The process about "seedling purchase (seedling propagation) - seafood cultivation - seafood fishing - seafood processing - seafood sales" is selected as the main line, and sensors are used to collect seafood information. The final information is pooled into the seafood traceability data center. Companies can view the seafood traceability data in the traceability system, and consumers can obtain product traceability data by scanning the two-dimensional code of product packaging. The system adopts B/S model and SSH framework to complete system development. The experimental results show that the new system provides powerful technical support for marine product traceability and marine product quality safety management. Based on the above finding, it is concluded that the traceability system has been technically able to effectively improve the product management of marine fishery enterprises through the information management technology. At the same time, it lays a good foundation for the overall upgrading and reform of China's marine fishery industry.
\end{abstract}

Keywords-Traceability system, Internet of things, marine products

\section{Introduction}

Compared with other kinds of food, seafood has the characteristics of variety and difference. A variety of microorganisms are very easy to breed in marine products, resulting in viruses, parasites and other biological parasites in the intestines, skin and muscles of seafood [1]. When people eat sick seafood, the probability of an incurable foodborne illness increases. Consumers' life and health are seriously affected by food safety problems caused by foodborne diseases [2]. Data statistics pointed out that the number of foodborne diseases in China reached three hundred million people each year. Because of these characteristics of seafood, seafood is more perishable during storage and transportation [3]. Therefore, it is necessary to strictly supervise the quali- 
ty and safety of marine products, and to grasp the quality and safety of marine products in real time. According to the real-time information, the quality and safety of seafood should be monitored and traced in a timely manner [4].

The food safety case in recent years has led to a more transparent understanding of how food is produced and transported. To realize the safety and quality of freshwater fish product traceability, the key point is to make sure the reasonable traceability unit, clear the responsibility body of the traceability system, ensure the validity of the retroactive information, and establish the software module of the traceability system. Because of the uniqueness of freshwater fish breeding, transportation and consumption, the traceability of freshwater fish products cannot be carried out effectively and completely, and the traceability unit is difficult to plan. This adds some difficulties to the establishment and development of traceability systems. Based on the above problems, a new aquatic product inspection system is established to improve existing insecurity factors.

\section{Literature review}

In order to solve the problem of seafood quality safety early warning, scholars at home and abroad have made a lot of research on the early warning methods of aquatic products, agricultural products and livestock products. There researches have achieved great results [5]. By contrast, overseas research on seafood safety early warning is relatively early. For example, there are several organizations such as the international seafood quality safety warning system of international food safety authorities network (INFOSAN), the global environment monitoring system (GEMS) of food contamination monitoring and assessment program (GEMS/Food), the EU rapid alert system for food and feed (RASFF) jointly organized by the WHO (WHO) and the food and agriculture organization of the United Nations (FAO), which are committed to the prevention of sudden outbreak of food safety incidents [6].

In the literature, the food safety early-warning system is studied from many perspectives, and the early warning system of food safety is constructed with the idea of system engineering [7]. AHP and support vector machines (SVM) are combined to predict the quality and safety of livestock products [8]. First of all, the importance of the evaluation index of livestock product quality and safety early warning is studied by AHP method, and then the early warning result is obtained by SVM pretreatment. In the literature, BP neural network is used to warn food safety according to monitoring data [9]. In the literature, BP neural network is combined with AHP to evaluate food safety, and AHP is used to evaluate indexes [10]. BP neural network training samples are used to obtain the quality and safety evaluation results [11]. A forewarning system of aquatic product safety is put forward in the literature [12]. Based on the comprehensive study of food safety early-warning theory and intelligence theory, a food safety intelligent early warning system is put forward [13]. 


\section{Method}

\subsection{Architecture design}

According to the data needed for each stage of marine aquaculture and marine aquaculture standards, traceability system collects aquaculture data at all stages of the product, such as feed, water exchange, pool time and other data [14]. Through the means of information, the electronic management is applied to the whole process of marine products from breeding to finished products to establish transparent identity files.

The system is built on the Windows 7 platform, while the B/S model and SSH framework are used to complete the background running. The back end adopts JAVA technology, and the front end mainly adopts JSP technology. The system generates traceability two-dimensional code according to the traceability data. Consumers read two-dimensional code information through mobile terminals. In addition, they can query and obtain relevant traceability data. After returning to the mobile terminal, the data is displayed in the mobile browser. It is conductive to improve user experience and traceability without installing redundant software. he management server records information such as IP addresses originating from the query, preventing hackers from accessing saturation servers. If the query execution is performed frequently by the same IP, the server automatically shields the IP for a period of time. For legitimate IP addresses that are authenticated through data interfaces, large data volumes are allowed to be continuously searched. However, the amount of access data per unit time is limited by the QoS policy. The system aims to finish the establishment of the batch of seafood. At the same time, the system should strengthen the research on rearing batch progress, breeding and processing and other related operations, to achieve traceability number.

\subsection{Traceability coding}

Traceability system uses MySQL database to store data. According to the demand of traceability system, information modules such as user management, culture container, batch status, feed feeding, aquaculture operation, fishing record, processing status and finished product logistics have been established.

The traceability coding based on the traceability data is the key link of the traceability system. The resulting traceability code consists of the following four components: product categories, coding, batch coding, production times and product numbers. The product category is produced according to the product category, which is stipulated by the enterprise itself. Batch coding requires traceability of product batch information. Batch coding takes the time when the product is started, the time of production is the date of production, and the product number is the product number of the product corresponding to the date of production. The aim is to show that the seafood is the product of the current production date. The coding chart is shown in figure 1. 


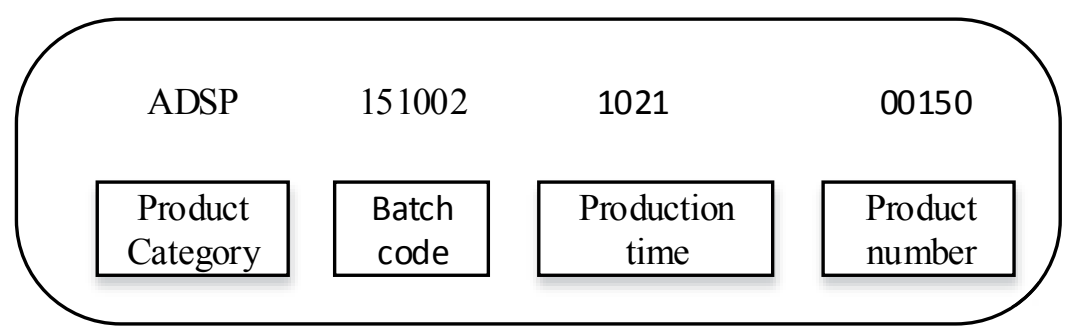

Fig. 1. Traceability coding schematic diagram

In order to ensure the safety of traceability of marine products, MD5 digital signature is used to generate the digital codes when the two-dimensional codes are generated. At present, there is no very effective way to crack MD5 encryption. Therefore, the encryption process can greatly guarantee the security of tracing source code and avoid imitations of counterfeit and shoddy products.

\subsection{System function}

Through the means of information, the marine traceability management system carries out the electronic management of the seafood from the seedling rearing to the finished product logistics and transportation. In view of the various stages of marine aquaculture, feeding records, culture containers, real-time inspection, and additions and deletions of aquaculture containers can be increased. In view of the fishing links, the fishing weight of fishing containers or the fishing weight are queried according to the date, so that users can control the production situation. In view of the processing stage of seafood, users can inquire about the processing stage and the time of logistics and transportation of the seafood. The specific functions are shown in figure 2.

Batch management is mainly used for new seafood batch, query each batch, control batch schedule. In the new batch, seafood companies may choose to buy sources or grow their own products. At the same time, users need to make a specific description for the source of purchase and culture so that it can be applied to a variety of seafood farming. The same batch of purchased seafood seedlings were placed in a number of seedling containers. The sensor obtains real-time water quality information, air humidity, growth status and so on. According to the data obtained by the sensors, the seedling managers observed the growth of marine products and analyzed the growth status of marine products. According to the growth of marine products, the management decided to put in the quantity and type of bait and whether to change water or change containers.

The management module of processing factory is mainly used for traceability management of seafood processing. Fresh seafood was imported into the factory and began to enter the processing plant. For different types of seafood, processing plants, production workers will carry out different production operations. At this point, the same batch of seafood began to enter different production lines, producing different seafood products. Two-dimensional code management module is mainly used for the management of two-dimensional code for user inquiry and traceability information. 
After the user generates a specified two-dimensional code instruction in the twodimensional code generation interface, the user monitors the background information of the sea products and sends it back to the source management server.

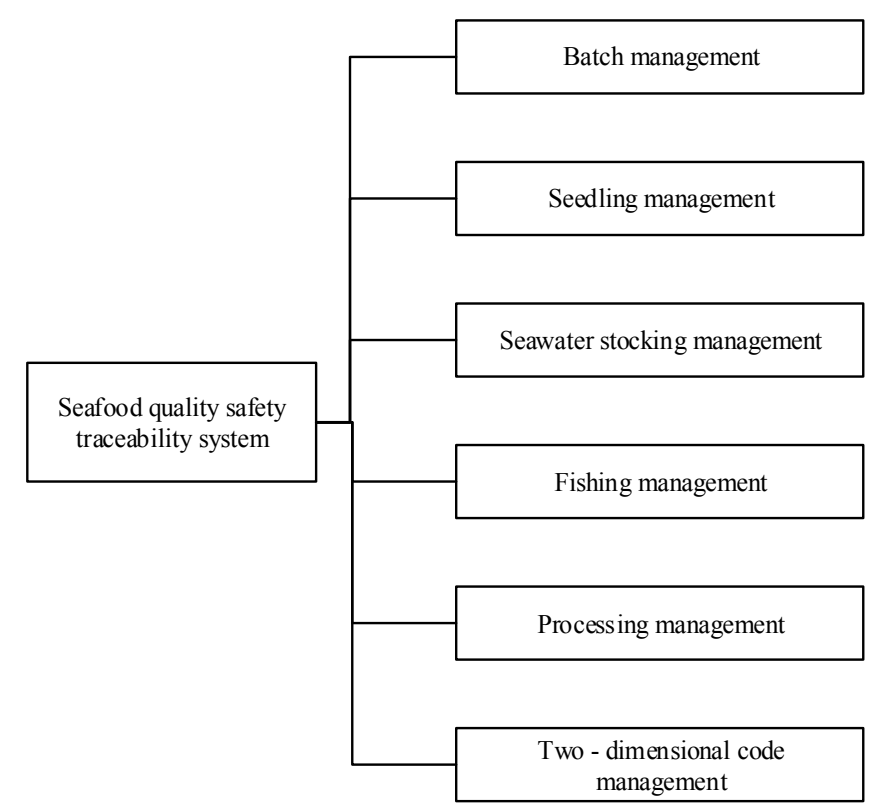

Fig. 2. System function diagram

\subsection{Internet of things}

The Internet of things is an information bearer based on the Internet and traditional telecommunication networks. It helps to realize the interconnection of all common physical objects that can be independently addressed. The traditional manual monitoring model of marine products is limited by the means of measurement, sampling frequency, sampling quantity, analysis efficiency, data processing and so on. It cannot respond to changes in seafood status in a timely manner. However, the use of Internet of things technology enables a variety of improvements. For example, the status of seafood has real-time monitoring of personnel, product data acquisition in real-time. In addition, the automatic control of breeding equipment, automatic adjustment of indoor temperature and humidity, seafood status display, statistical analysis, and so on can also be realized.

The traceability system can deploy a large number of low cost small wireless sensors in the monitored area. The sensor nodes have four modules: perception, calculation, communication, and battery. The nodes form a self-organizing network via wireless channels and transmit the perceived data to the sink node in a timely and efficient manner. The sink node further submits data to the Internet for use in the upper layer. At the same time, commands from the Internet can also reach each sensor in the network through the sink node. 


\section{$4 \quad$ Result and discussion}

\subsection{Key technology}

In the seafood safety traceability system, the system framework is established based on the bastard seedling purchases and seafood sales. The key of the system is to collect, track and record all kinds of information about the whole period of the product. The completeness and accuracy of information and how to define the applicability of data are important signs that traceability systems need to focus on. Compared with other crops such as agricultural products, the difference is that seafood needs to be farmed or caught directly into the sea. Therefore, it is necessary to consider a new way of tracking, and the use of Internet of things technology can greatly enhance the feasibility of traceability of seafood activities. Therefore, the system will achieve a seafood integration traceability system for the specific seafood by using the Internet of things technology, MySQL database and SSH framework.

\subsection{System framework}

The traceability system adopts the B/S technology framework, and SSH (Spring+Structs2+Hibernate) is taken as the programming framework. The system framework is shown in figure 3. SSH is a popular JavaWEB open source framework at present.

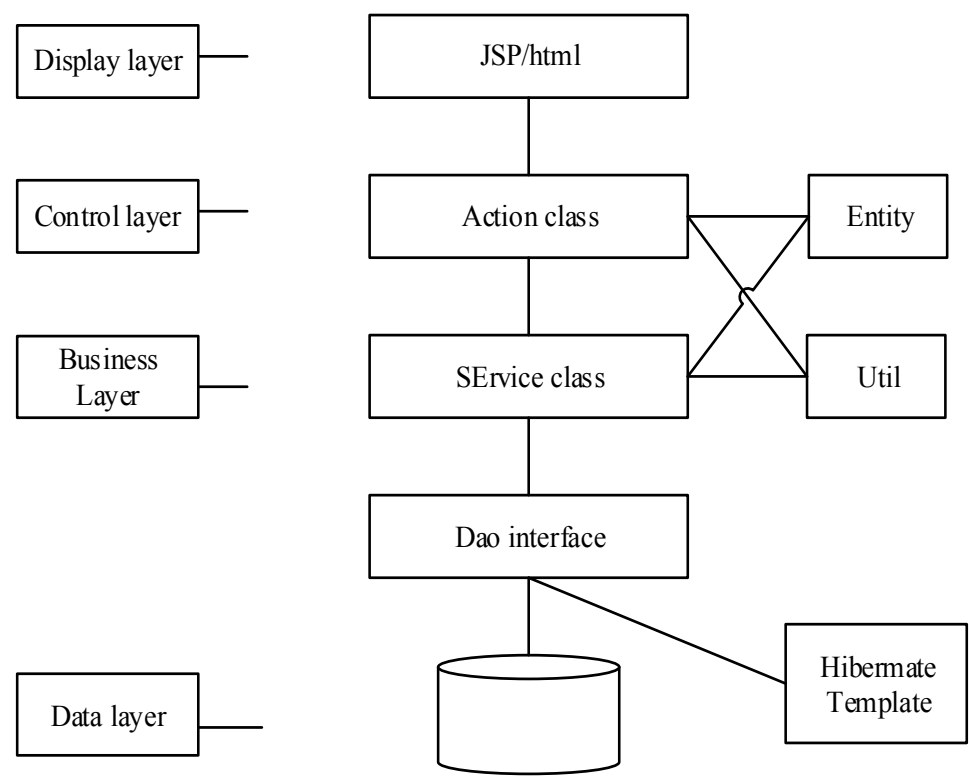

Fig. 3. System architecture diagram 
The SSH framework is divided into five layers from the responsibility, which is domain object layer, data access object layer, business logic layer, controller layer and presentation layer. Each layered component is not coupled in a hard-coded manner, so the hierarchical architecture facilitates the scalability of the platform. Among them, as the overall infrastructure of the system, Struts is responsible for the separation of MVC. In the model section of the Struts framework, the Hibernate framework is used to support the persistence layer and the business layer is supported by Spring. The specific approach is to establish some model based on object-oriented analysis method, such as the JavaBean entity. Hibernate is used to implement these basic DAO (Data, Access, Object) interfaces. The Hibernate related configuration is adopted, which enables entity classes to be associated with data tables in the database, enabling conversions and access between classes and data. Finally, the business logic of the whole system is unified by Spring.

\section{Conclusion}

China is a big fishing country. Fishery plays more and more important role in the country's foreign trade and economy. Internet of things technology has been used in seafood farming. It can ensure the transparency of the data of various aspects such as culture, harvesting, processing and transportation of marine products. It provides powerful technical support for marine product traceability and marine product quality safety management. The marine product traceability system based on the Internet of things technology can greatly improve the quality management ability, logistics management ability and competitiveness of domestic and international trade in the process of seafood traceability.

The traceability system has been technically able to effectively improve the product management of marine fishery enterprises through the information management technology. At the same time, it lays a good foundation for the overall upgrading and reform of China's marine fishery industry. However, it still restricts the development of traceability system because of the diversity of the traceability methods and the coupling between the Internet of things and the traceability system. These issues will be traced to the system for further research and improvement directions.

\section{References}

[1] Zhou, J., \& Jin, S. (2015). An empirical analysis of the implementation of vegetable quality and safety traceability systems centering on wholesale markets. Journal of Physics Condensed Matter, 18(1), págs. 205-220. Https://doi.org/10.1142/9789814447768.

[2] Kang, Y. S., Park, I. H., \& Youm, S. (2016). Performance prediction of a mongodb-based traceability system in smart factory supply chains. Sensors, 16(12), 2126. Https://doi.org/10.3390/s16122126.

[3] Jakkhupan, W., Arch-Int, S., \& Li, Y. (2015). An rfid-based traceability system. Telecommunication Systems, 58(3), 243-258. Https://doi.org/10.1007/s11235-014-9866-7. 
[4] Lu, C., Wang, B., Peng, X. Y., Hou, X. L., Bai, B., \& Wang, C. M. (2015). Study of intelligent agricultural cultivation management plan model based on geographic information system. Open Biotechnology Journal, 9(1), 150-156. Https://doi.org/10.2174/1870707 01610010390.

[5] Yongsawatdigul, J., Wongngam, W., \& Khampirat, B. (2016). Chemical parameters for traceability of raw material freshness of tropical surimi. Journal of Aquatic Food Product Technology, 29(4), 815-827. Https://doi.org/10.1080/10498850.2014.964433.

[6] Aiello, G., Enea, M., \& Muriana, C. (2015). The expected value of the traceability information. European Journal of Operational Research, 244(1), 176-186. Https://doi.org/10.1016/j.ejor.2015.01.028.

[7] Dubovikov, N. I., Podmurnaya, O. A., Skryabikov, N. P., Sokov, I. A., \& Vinge, A. F. (2016). The russian national standard of gases humidity and traceability system of humidity measurements. International Journal of Thermophysics, 37(5), 1-12. Https://doi.org/10.1007/s10765-015-2014-0.

[8] Efremov, S., Pilipenko, N., \& Voskov, L. (2015). An integrated approach to common problems in the internet of things. Procedia Engineering, 100(3), 1215-1223. Https://doi.org/10.1016/j.proeng.2015.01.486.

[9] Der, P., \& Egyed, A. (2015). Do developers benefit from requirements traceability when evolving and maintaining a software system?. Empirical Software Engineering, 20(2), 413441. Https://doi.org/10.1007/s10664-014-9314-z.

[10] Wang, Y., Tan, R., Xing, G., Wang, J., Tan, X., \& Liu, X., et al. (2016). Monitoring aquatic debris using smartphone-based robots. IEEE Transactions on Mobile Computing, 15(6), 1412-1426. Https://doi.org/10.1109/tmc.2015.2460240.

[11] Fan, K., Li, H., \& Yang, J. (2015). Traceability analysis and coupling compensation for guideway-induced errors in large cnc gantry guideway grinder. International Journal of Advanced Manufacturing Technology, 80(5-8), 907-919. Https://doi.org/10.1007/s00170015-7070-x.

[12] Bünger, L., Anhalt, K., Taubert, R. D., Krüger, U., \& Schmidt, F. (2015). Traceability of a ccd-camera system for high-temperature measurements. International Journal of Thermophysics, 36(8), 1784-1802. Https://doi.org/10.1007/s10765-015-1915-2.

[13] Pérez, M. M., González, G. V., \& Dafonte, C. (2016). Evaluation of a tracking system for patients and mixed intravenous medication based on rfid technology. Sensors, 16(12), 2031. Https://doi.org/10.3390/s16122031.

[14] Meyer, David, Prien, Ralf, D., Dellwig, \& Olaf, et al. (2016). Sensors, vol. 16, pages 2027: a multi-pumping flow system for in situ measurements of dissolved manganese in aquatic systems. Sensors, 16(12), 2027. Https://doi.org/10.3390/s16122027.

\section{$7 \quad$ Author}

Xiao-hua Xu is with Tianjin Agricultural University, Tianjin 300384, China (xuxiaohua@tjau.edu.cn).

Article submitted 14 August 2017. Published as resubmitted by the author 19 September 2017. 